\title{
O papel de Ancyrocephalinae (Monogenea: Dactylogyridae), parasito de Geophagus brasiliensis (Pisces: Cichlidae), como indicador ambiental
}

\author{
The role of Ancyrocephalinae (Monogenea: Dactylogyridae), parasite of \\ Geophagus brasiliensis (Pisces: Cichlidae), as an environmental indicator \\ Rubens Riscala Madi*; Marlene Tiduko Ueta
}

Departamento de Parasitologia, Instituto de Biologia, Universidade Estadual de Campinas - UNICAMP

Recebido em 5 de Dezembro de 2006

Aprovado em 22 de Fevereiro de 2009

\section{Resumo}

As variaçôes nas populaçóes de parasitos em peixes podem ser indicadoras de alguma alteração ambiental. Tendo por objetivo o estudo da variação do parasitismo por Ancyrocephalinae em Geophagus brasiliensis, foram comparados dois reservatórios de caracterizaçôes tróficas distintas (reservatórios do Juqueri - eutrófico - e do Jaguari - oligomesotrófico). Para isso, foram medidas, bimestralmente, as taxas de prevalência e intensidade de infecçâo deste parasita ao longo de um ano. Os resultados demonstraram que a prevalência e a intensidade de infecção variam conforme ocorre a alteração do nível de armazenamento dos reservatórios e, consequentemente, as variaçóes da quantidade de material em suspensão na água.

Palavras-chave: Parasitismo de peixes, prevalência, intensidade de infecção, turbidez.

\begin{abstract}
The variations in the parasite population of fishes may be indicative of environmental alterations. With the aim of studying the variation of the parasitism by Ancyrocephalinae in Geophagus brasiliensis, two reservoirs of distinct trophic caracterizations (Juqueri - eutrophic and Jaguari - oligomesotrophic) were compared. The prevalence rates and intensity of infection of this parasite were measured bimonthly for one year. The results showed that the prevalence and the intensity of infection vary in according to the alteration in the storage level of the reservoirs and consequently to the variations in the amount of material in suspension in the water.
\end{abstract}

Keywords: Fish parasites, prevalence, intensity of infection, turbidity.

\section{Introduçáo}

Tendo os parasitos como indicadores da biologia do hospedeiro, de contaminantes ambientais e estrutura de cadeia alimentar, a comunidade parasitária de peixes pode ser um bom demonstrativo de estresse ambiental e biodiversidade (CHUBB, 1980, 1982; OVERSTREET, 1997). Bons indicadores podem ser excepcionalmente sensíveis às modificaçôes ambientais, e as alteraçóes significativas no número de indivíduos nas populaçóes podem ser utilizadas como um alerta das condiçóes deteriorantes, antes da maioria dos organismos menos sensíveis serem seriamente afetados (MACKENZIE et al., 1995)

$\mathrm{O}$ ciclo de alguns parasitos depende de organismos pequenos e delicados e a mínima variação ambiental atinge esses organismos, refletindo sobre o parasitismo dos peixes. A escolha de qual parasito

\footnotetext{
*Autor para correspondência: Rubens Riscala Madi

Departamento de Parasitologia, Instituto de Biologia, Universidade Estadual de Campinas - UNICAMP, Cidade Universitária Zeferino Vaz, s/n, CP 6109, CEP 13083-970 Campinas - SP, Brasil; e-mail: rmadi@unicamp.br Este trabalho é parte da tese de doutorado defendida no Programa de Pós-graduação em Parasitologia da UNICAMP
}

apresenta condições de ser utilizado como ferramenta de análise ambiental é complexa.

A utilizaçâo de monogenéticos como indicadores de alteraçôes ambientais foi citada por Mackenzie et al. (1995), que afirmaram que o baixo nível de poluição pode produzir um efeito hormético em alguns ectoparasitas, aumentando a reprodução e causando um aumento marcante nas populaçôes parasitas.

Os membros da classe Monogenea são ectoparasitos de brânquias, pele e orifícios de peixes, anfíbios e répteis e, menos frequentemente, do trato esofagiano e vesícula (CHUBB, 1977; EUZET, COMBES, 1998; BAKKE et al., 2002). Calcula-se que há mais de 3.000 espécies de monogenéticos parasitas de peixes (EIRAS, 1994), muitos dos quais específicos para determinada espécie hospedeira (BAKKE et al., 2002.).

Para o presente trabalho, foi selecionado como peixe hospedeiro o Geophagus brasiliensis (Cichlidae; Perciformes), segundo critérios elaborados por Overstreet (1997) e corroborados por Sures 
(2004) - peixes com restrito "home range", comum, abundante, de fácil amostragem, capazes de servir como hospedeiro para um número relativamente grande de espécies parasitas, além de apresentar tamanho relativamente pequeno - tendo por objetivos comparar as variaçóes na prevalência e na intensidade de infecção por monogenéticos Ancyrocephalinae, em dois reservatórios de caracterizaçóes tróficas distintas, para analisar a viabilidade da utilização deste parasito como indicador ambiental.

\section{Material e Métodos}

Foram realizadas coletas bimestrais entre setembro de $2001 \mathrm{e}$ setembro de 2002, nos reservatórios do Juqueri (23 $19^{\prime} 07^{\prime \prime} \mathrm{S}$ e $46^{\circ} 35^{\prime} 12^{\prime \prime} \mathrm{W}$, altitude $745 \mathrm{~m}$ - eutrófico) e do Jaguari (22 $53^{\prime} 20^{\prime \prime} \mathrm{S}$ e 462 24' 49" W, altitude 845 m - oligomesotrófico), Estado de São Paulo. Ambos os reservatórios foram criados para a captação de água para abastecimento e pertencem ao Sistema Cantareira sob administração da Sabesp (Companhia de Saneamento Básico do Estado de São Paulo). Distam aproximadamente $100 \mathrm{~km}$ um do outro e estão interligados por túneis e canais através dos reservatórios do Jacareí, Cachoeira e Atibainha (Figura 1).

Os peixes foram capturados utilizando-se redes de espera de malhagens variadas, colocadas, a partir da margem, ao entardecer e retiradas na manhã do dia seguinte.

Foram medidas temperatura, taxa de oxigênio dissolvido, $\mathrm{pH}$, turbidez e condutividade da água de todos os pontos de coleta, utilizando-se equipamentos de multianálise de água. A avaliação da transparência da água foi realizada utilizando-se um disco de Secchi de $20 \mathrm{~cm}$ de diâmetro.

Os exemplares de $G$. brasiliensis coletados foram pesados, medidos (comprimento total), e as brânquias foram isoladas inteiras e mergulhadas em frascos com solução de formol 1:4.000 por uma hora, submetidas a agitaçóes em intervalos regulares. Em seguida, a solução foi colocada em placa de Petri pequena, e os monogenéticos presentes foram contados e fixados em formol $5 \%$, sendo alguns exemplares corados pelo Tricrômico de Gomori, e outros montados em meio de Hoyer (PUTZ; HOFFMAN, 1963; KRITSKY et al., 1995; EIRAS et al., 2000).

Os dados de prevalência e intensidade de infecção foram obtidos seguindo os conceitos propostos por Bush et al. (1997).
Para as análises estatísticas dos resultados, foi realizado o teste de comparação múltipla de Duncan para avaliar as diferenças de prevalências totais e de intensidades de infecção entre os reservatórios e na comparação das variações das prevalências e das intensidades de infecção nas coletas, para cada reservatório. O teste "U" de Mann-Whitney foi empregado para a comparação das variaçóes das prevalências e das intensidades de infecção entre as coletas e entre os reservatórios. $\mathrm{O}$ coeficiente de correlação de Pearson foi utilizado para análise da correlação da prevalência e da intensidade de infecção com o peso e comprimento do peixe hospedeiro e com os fatores ambientais (temperatura, $\mathrm{pH}$, oxigênio dissolvido, turbidez, condutividade, transparência da água medida pelo disco de Secchi).

Quando necessário, os dados de intensidade de infecção foram logaritimizados. Todos os testes foram aplicados com intervalo de confiança igual a 5\%. As análises estatísticas foram realizadas com auxílio dos programas estatísticos SAS (SAS Inc., 1996) e BioEstat 2.0 (AYRES et al., 2000).

As médias dos índices de armazenamento do Sistema Cantareira foram obtidas na Sabesp (Companhia de Saneamento Básico do Estado de São Paulo, 2002).

\section{Resultados}

Foram examinados 550 exemplares de $G$. brasiliensis, sendo 280 do reservatório do Jaguari e 270 do reservatório do Juqueri.

Monogenéticos pertencentes à subfamília Ancyrocephalinae foram encontrados nas brânquias de G. brasiliensis em 76,79\% dos exemplares examinados no reservatório de Jaguari, com média de $5,51( \pm 6,35)$ vermes/peixe e em $82,22 \%$ no reservatório do Juqueri, apresentando, em média, $6,02( \pm 6,52)$ vermes/peixe. A prevalência total e a intensidade de infecção não diferiram entre os reservatórios do Jaguari $(\mathrm{p}=0,12)$ e do Juqueri $(\mathrm{p}=0,07)$.

Ainda comparando-se os reservatórios, porém levando-se em consideração a variação entre as coletas, a variação da prevalência (Figura 2a) e a variação da intensidade de infecção (Figura 2b), foram similares ao teste " $U$ " de Mann-Whitney $p=0,34$ e $p=0,15$, respectivamente. Mas, analisando-se a variação entre as coletas, dentro de cada reservatório, a prevalência foi diferente ao teste de Duncan, tanto no reservatório do Jaguari quanto no de Juqueri

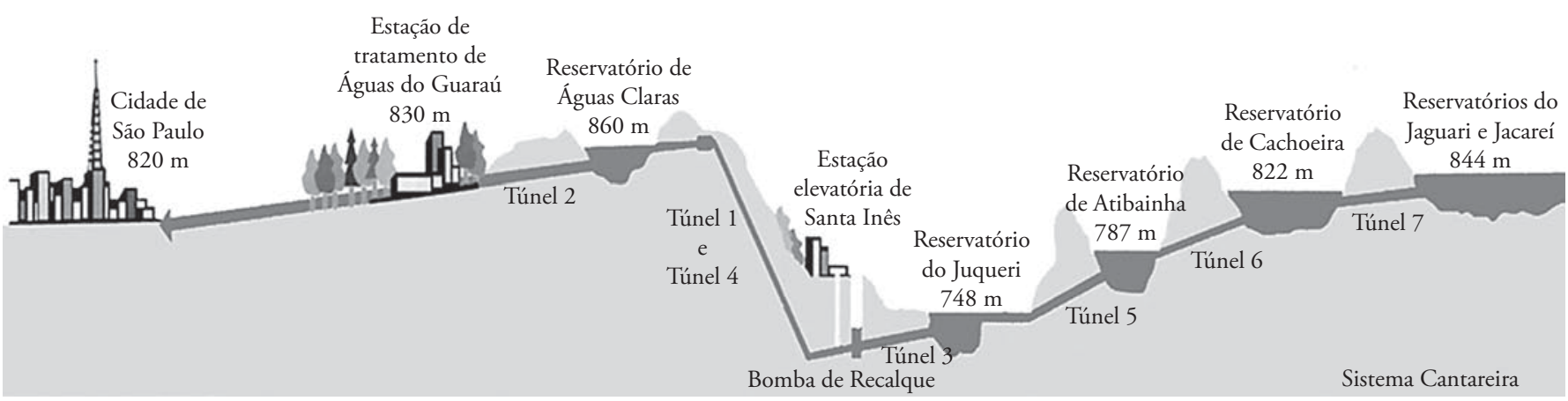

Figura 1. Perfil do Sistema Cantareira (Fonte: Sabesp). 

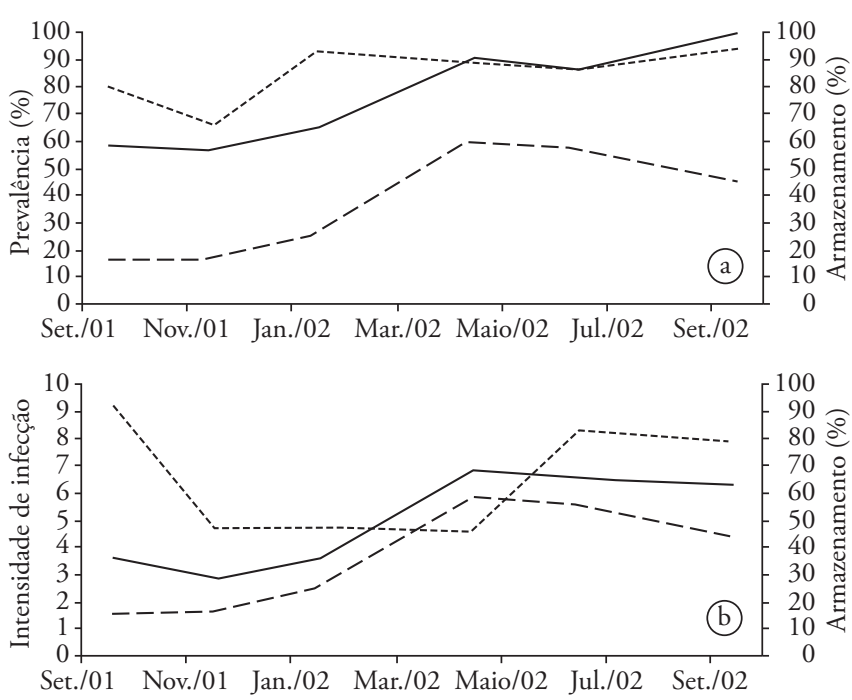

- Reservatório do Jaguari ------ Reservatório do Juqueri - - - Armazenamento do Sistema Cantareira

Figura 2. Variação da prevalência a) e da intensidade de infecção; e b) de Ancyrocephalinae em Geophagus brasiliensis, nos reservatórios do Juqueri e Jaguari, entre setembro de 2001 e setembro de 2002.

$(\mathrm{p}<0,01)$. O mesmo ocorreu para a variação na intensidade de infecção $(\mathrm{p}<0,01)$.

Nenhum parâmetro, tanto biológico quanto ambiental, correlacionou significativamente com a prevalência e com a intensidade de infecção.

\section{Discussáo}

A influência da fragmentação sobre a comunidade parasitária de peixes, em lagos com diferentes características eutróficas e níveis de poluição, foram estudadas por Valtonen et al. (1997). Quando comparararam um lago oligotrófico e despoluído com outros dois eutrofizados e poluídos, encontraram altas prevalências de Dactylogyrus sp. nos lagos poluídos, atribuídas por estes autores como decorrentes da queda de imunidade do peixe hospedeiro em virtude da poluição química. Ambientes eutrofizados ou com níveis de turbidez elevados devido ao aumento de material em suspensão na água ou poluição, mesmo em diferentes graus, possibilita uma maior ocorrência de irritação nos filamentos branquiais dos peixes, aumentando, assim, a susceptibilidade aos parasitos como os monogenéticos (SKINNER, 1982). Khan e Kiceniuk (1988) também sugeriram que a irritação das brânquias por fraçóes hidrossolúveis de óleo, resultando em hiperplasia das células epiteliais acompanhada por excessiva secreçáo de muco e coagulação, talvez produzam um habitat que contribua para a infestação e reprodução dos parasitos. Moles e Wade (2001), em observação experimental, reportaram que a exposição do peixe Ammodytes hexapterus ao sedimento contaminado por hidrocarboneto de petróleo (PHC), por 90 dias, reduz a atividade fagocítica dos macrófagos e foi associada com um aumento significativo na abundância e prevalência de Gyrodactylus sp., em relação ao grupo controle. Estes autores concluíram que as concentraçóes de PHC foram suficientes para deprimir o sistema imune do peixe, provocando um aumento do parasitismo.

Em vista disso, era de se esperar que a prevalência de Ancyrocephalinae em $G$. brasiliensis, no reservatório do Juqueri (eutrófico e "poluído"), fosse significativamente maior que no reservatório do Jaguari (oligomesotrófico e despoluído). O fato da prevalência dos parasitos deste grupo entre os reservatórios (Figura 2a) não diferir estatisticamente, e a elevação da intensidade de infecção no reservatório do Jaguari (Figura 2b), pode ser explicado pela estiagem ocorrida em meados de 2001. O reservatório do Jaguari reduziu expressivamente sua capacidade de armazenamento, permanecendo, assim, até aproximadamente o mês de novembro do mesmo ano, quando começaram efetivamente as chuvas na região. Durante a época de chuvas, ocorreu um fluxo maior de terra, e de outras cargas difusas (plantas, sementes, fertilizantes, defensivos agrícolas, etc.), foram arrastadas pelas enxurradas, provenientes das propriedades às margens do reservatório, que fez com que, nesse período, houvesse um aumento do nível de material em suspensão na água. Ao mesmo tempo, enquanto ocorria a elevação do nível de água no reservatório do Jaguari, os reservatórios subsequentes do Sistema Cantareira receberam as águas provenientes das próprias bacias de drenagem, sem grandes alteraçóes nos seus níveis de armazenamento. O reservatório do Juqueri, recebendo em pouco tempo um volume de água relativamente alto do reservatório do Atibainha que, de acordo com Salas e Martino (1991) possui características oligotróficas, provavelmente alterou sutilmente o seu grau de eutrofização, afetando a intensidade de infecção por Ancyrocephalinae presentes em G. brasiliensis. Ao final da estação de chuvas, a intensidade de infecção, que depois de diminuir estava estabilizada, tornou a aumentar, voltando quase ao índice obtido no início do trabalho.

Apesar das medidas epidemiológicas, neste trabalho, terem sido realizadas em condiçóes atípicas, as mudanças ocorridas no índice de armazenamento do Sistema Cantareira, refletidas na prevalência e na intensidade de infecção por Ancyrocephalinae em $G$. brasiliensis, leva a supor que este parasita atue como indicador de alteração ambiental.

\section{Agradecimentos}

Os autores agradecem à Sabesp (Companhia de Saneamento Básico do Estado de São Paulo), ao Grupo de Pesquisa em Ecossistemas Aquáticos Sujeitos a Impactos Ambientais da PUC Campinas, a João Batista Alves de Oliveira, ao Maurício Solera Rodrigues da Silva e ao Prof. Dr. Arício Xavier Linhares.

\section{Referências}

AYRES, M. et al. BioEstat 2.0: aplicações estatísticas nas áreas de ciências biológicas e médicas. Belém: Sociedade Civil Mamirauá/MCT-CNPQ, 2000. 272 p.

BAKKE, T. A.; HARRIS, P. D.; CABLE, J. Host specificity dynamics: observations on gyrodactylid monogeneans. International Journal for Parasitology, v. 32, n. 3, p. 281-308, 2002. 
BUSH, A. O. et al. Parasitology meet ecology on its own terms: Margolis et al. revisited. Journal of Parasitology, v. 83, n. 4, p. 575-583, 1997.

CHUBB, J. C. Seasonal ocurrence of helminths in freshwater fishes. Part I. Monogenea. Advances in Parasitology, v. 15, p. 133-199, 1977.

CHUBB, J. C. Seasonal ocurrence of helminths in freshwater fishes. Part III. Larval Cestoda and Nematoda. Advances in Parasitology, v. 18 , p. 1-120, 1980.

CHUBB, J. C. Seasonal ocurrence of helminths in freshwater fishes. Part IV. Adult Cestoda, Nematoda and Acanthocephala. Advances in Parasitology, v. 20, p. 1-292, 1982.

COMPANHIA DE SANEAMENTO BÁSICO DO ESTADO DE SÃO PAULO - SABESP. Situação dos Mananciais. Disponível em: <http://www.sabesp.com.br>. Acesso em: Agosto de 2001 e Novembro de 2002.

EIRAS, J. C. Elementos de Ictioparasitologia. Porto: Fundação Eng. António Almeida, 1994. 339 p.

EIRAS, J. C.; TAKEMOTO, R. M.; PAVANELLI, G. C. Métodos de estudo e técnicas laboratoriais em parasitologia de peixes. Maringá: Editora Universidade Estadual de Maringá, 2000. 173 p.

EUZET, L.; COMBES, C. The selection of habitats among the monogenea. International Journal for Parasitology, v. 28, n. 10, p. $1645-1652,1998$.

KHAN, R. A.; KICENIUK, J. W. Effect of petroleum aromatic hydrocarbons on monogenoids parasitizing Atlantic cod, Gadus morhua L. Bulletin of Environmental Contamination and Toxicology, v. 41, n. 1, p. 94-100, 1988.

KRITSKY, D. C.; BOERGER, W. A.; POPAZOGLO, F. Neotropical Monogenoidea. 22. Variation in Scleroductus species (Gyrodactylidea, Gyrodactylidae) from Siluriform fishes of southeastern Brazil. Journal of the Helminthological Society of Washington, v. 62, n. 1, p. 53-56, 1995.

MACKENZIE, K. et al. Parasites as indicators of water quality and the potential use of helminth transmission in marine pollution studies. Advances in Parasitology, v. 35, p. 85-144, 1995.

MOLES, A.; WADE, T. L. Parasitism and phagocytic function among sand lance Ammodytes hexapterus Pallas exposed to crude oil-laden sediments. Bulletin of Environmental Contamination and Toxicology, v. 66, n. 4 , p. 528-535, 2001.

OVERSTREET, R. M. Parasitological data as monitors of environmental health. Parassitologia, v. 39, n. 3, p. 169-175, 1997.

PUTZ, R. E.; HOFFMAN, G. L. Two new Gyrodactylus (Trematoda, Monogenea) from cyprinid fishes with synopsis of those found on North American fishes. Journal of Parasitology, v. 49, n. 4, p. 559, 1963.

SALAS, H. J.; MARTINO, P. A simplified phosphorus trophic state model for warm-water tropical lakes. Water Research, v. 25, n. 3, p. 341-350, 1991.

SAS INSTITUTE INCORPORATION. SAS User's Guide: statistics. Release 6.12. North Caroline, Cory, 1996. 1098 p.

SKINNER, R. H. The interrelation of water quality, gill parasites, and gill pathology of some fishes from south Biscayne Bay, Florida. Fishery Bulletin, v. 80, n. 2, p. 269-280, 1982.

SURES, B. Environmental parasitology: relevancy of parasites in monitoring environmental pollution. Trends in Parasitology, v. 20, n. 4 , p. $170-177,2004$.

VALTONEN, E. T.; HOLMES, J. C.; KOSKIVAARA, M. Eutrophication, pollution and fragmentation: effects on the parasite communities in roach and perch in four lakes in Central Finland. Parassitologia, v. 39, n. 3, p. 233-236, 1997. 